ISSN: $2317-8957$

Volume 6, Number 1, Jun. 2018

\title{
BIOFIXAÇÃO DE CO2 PELA MICROALGA MONORAPHIDIUM SP.
}

\author{
YORDANKA REYES CRUZ ${ }^{1}$, ANDREINA Z. FIGUERA LEONETT ${ }^{1}$, GISEL CHENARD DÍAZ ${ }^{1}$, RENÉ \\ GONZALEZ CARLIZ ${ }^{1}$, VINICIUS ROSSA ${ }^{1}$, MARINA GALINDO ${ }^{2}$, DONATO ALEXANDRE GOMES \\ ARANDA $^{1}$, LUCIANO BASTO OLIVEIRA ${ }^{3}$. \\ ${ }^{1}$ Escola de Química, Universidade Federal do Rio de Janeiro (UFRJ), RJ, Brasil. \\ ${ }^{2}$ Universidade Agrícola de Havana (UNAH), Havana, Cuba. \\ ${ }^{3}$ Instituto Virtual Internacional de Mudanças Globais (IVIG/COPPE/UFRJ), RJ, Brasil.
}

\begin{abstract}
RESUMO
As microalgas são organismos unicelulares capazes de realizar fotossíntese, aproveitando a luz solar e absorvendo CO2 para sintetizar a própria biomassa. Exatamente por esta característica, elas têm sido visadas nosúltimos anos por diferentes setores, especialmente pela capacidade de reduzir as emissões de $\mathrm{CO} 2$ e para em paralelo sintetizar biomassa que pode ser utilizada na produção de biocombustíveis. Além de serem microrganismos de crescimento rápido, possuem uma composição bioquímica diversificada. Neste contexto o presente trabalho teve como objetivo avaliar a biofixação de $\mathrm{CO} 2$ pela microalga Monoraphidium sp., cultivada em fotobiorreator fechado tipo Janela, e a caracterização da biomassa microalgal produzida em relação ao: teor de lipídios totais - LT, lipídios convertíveis em biodiesel - LCB, carboidratos e proteínas. Os resultados permitiram definir que o melhor resultado foi obtido após $24 \mathrm{~h}$ de cultivo, no qual, por cada grama de biomassa, consumiram-se aproximadamente 1,2g de CO2. Abiomassa de Monoraphidium sp. produzida apresentou um teor de LT de $17,37 \pm 3,27 \%$, LCB de aproximadamente $8,36 \pm 2,69 \%$, carboidratos de $32 \pm 3,37 \%$ e proteínas de $34,26 \pm 0,41 \%$.
\end{abstract}

Palavras-chave: Biomassa, Captura de CO2, Cultivo, Fotobiorreator, Microalgas.

\begin{abstract}
Microalgae are unicellular organisms capable of photosynthesis, turning sunlight and carbon dioxide (CO2) into rich biomass. Precisely because of this definition, in recent years various sectors have been targeting their ability to reduce $\mathrm{CO} 2$ emissions and the capacity of simultaneously synthesize biomass which can be later used to produce bio-fuels. Besides being considered fast-growth microorganisms, microalgae have a diverse biochemical composition with similar characteristics to traditional biomass. In this context, the present work aimed to evaluate the biofixation of $\mathrm{CO} 2$ by the microalgae Monoraphidium sp., cultivated in a closed-window type photobioreactor, as well as characterization of microalgal biomass produced in relation to the total lipid content (TL), lipids converted into biodiesel (LCB), carbohydrates and proteins. The results achieved showed that the best result was obtained after 24 hours of cultivation, where for each gram of biomass produced approximately $1,2 \mathrm{~g}$ of CO2 were consumed. The biomass Monoraphidium sp. produced had a content of lipids converted into biodiesel of approximately $8.36 \pm 2.69 \%$, carbohydrates $32 \% \pm 3.37$ and proteins $34.26 \% \pm 0.41$.
\end{abstract}

Keywords: Biomass, $\mathrm{CO} 2$ capture, Cultivation, Microalgae, Photobioreator.

\section{INTRODUÇÃO}

Microalgas são organismos unicelulares, fotossintetizantes, capazes de utilizar energia luminosa com maior eficiência que as plantas superiores e são excelentes fixadoras de gás carbônico (Greenwell et al., 2010).
Em princípio, as microalgas foram investigadas como fonte de obtenção de proteína para o consumo humano. O foco inicial deslocou-se para seu uso na captura de $\mathrm{CO} 2$ e para em paralelo aproveitar a biomassa sintetizada na produção de biocombustíveis, tais como: hidrocarbonetos renováveis (bioquerosene 
ou diesel verde), biodiesel ou etanol de segunda geração, alem de vários outros produtos, como hidrogênio, produtos alimentícios, farmacêuticos e cosméticos (Greenwell et al, 2010; Perlingeiro et al., 2014).

Dentre as características das microalgas, destaca-se o fato de crescerem rapidamente, sintetizar e acumular grandes quantidades de lipídios, carboidratos, proteínas e pigmentos, como os carotenóides e a clorofila. A fim de aproveitar ao máximo a cultura de microalgas é essencial a seleção da cepa adequada e das melhores condições de cultivo. Condições de cultivo envolvem: qualidade da água; $\mathrm{pH}$; temperatura; nutrientes e dosagem de $\mathrm{CO} 2$ de maneira controlada. Nutrientes devem ser adicionados para garantir o crescimento das microalgas (nitrogênio, fósforo, minerais e vitaminas), além de uma fonte de carbono (CO2) e energia solar.

O cultivo fototrófico é o mais utilizado, principalmente na produção em larga escala. A variação de lipídios nesta condição está entre 5 e $60 \%$, de acordo com o tipo de microalga (Mata et al., 2010). Este tipo de cultivo emprega iluminação, como fonte de energia, e carbono inorgânico $(\mathrm{CO} 2)$, proveniente do ar atmosférico ou injetado de cilindros pressurizados, como fonte de carbono para produzir energia química através do processo de fotossíntese. Nos sistemas fechados a eficiência da remoção e fixação de $\mathrm{CO} 2$ depende da espécie de microalga, da concentração de $\mathrm{CO} 2$, do "design" do fotobiorreator e das condições de operação (Raven et al., 2010).

No Brasil a microalga Monoraphidium sp. é cultivada no nordeste do pais, na cidade de Natal. Dentre as técnicas de produção em larga escala destaca-se o uso de raceway, que são grandes tanques abertos (cultivadores da planta piloto de Petrobras/UFRN em Extremoz/ $\mathrm{RN})$. Também, a usina de biodiesel Grande Valle BioEnergia Ltda., localizada no estado do Rio de Janeiro, em conjunto com a Fundação de Amparo à Pesquisa do Estado do Rio de Janeiro - FAPERJ, fez alguns investimentos no desenvolvimento de tecnologia para o cultivo e uso da microalga Monoraphidium sp. como matéri- a-prima alternativa na produção de biodiesel (EBC, 2013).

São poucos os estudos publicados relacionados com a captura de dióxido de carbono (CO2) pela microalga Monoraphidium sp. A maioria dos estudos estão focados na produção de biodiesel. Shrivastav et al., (2015) observaram que na adição de $\mathrm{CO} 2$ e ar, a microalga Monoraphidium sp. tem capacidade para mitigar o $\mathrm{CO} 2$, devido a sua alta capacidade de fixar carbono. E, em decorrência de sua alta produtividade de lipídios e biomassa, representa uma matéria-prima potencial para biocombustíveis. Apesar da espécie Monoraphidium sp. se adaptar muito bem a regiões de baixa temperatura e de pouca incidência luminosa, apresentando bom crescimento em águas residuais e com teores lipídicos semelhantes ao da soja (Holbrook et al., 2014), a Monoraphidium sp. é capaz de crescer em temperatura de $35^{\circ} \mathrm{C}$ (Report, 1987).

De acordo com a literatura, um quilograma de biomassa seca de microalga utiliza aproximadamente 1,83 quilogramas de $\mathrm{CO} 2$ (Brennan e Owende, 2010). Estudos têm sido desenvolvidos para validar as microalgas como biofiltros com o objetivo de purificar gases de combustão provenientes das principais indústrias geradoras como: termelétricas, cimenteiras, agropecuária, e outras. Assim, as microalgas estão sendo utilizadas para melhorar a qualidade do biogás pela redução do teor de $\mathrm{CO} 2$, o quê levaria a um aumento na concentração de metano (CH4).

Apesar de que há ainda uma série de desafios tecnológicos a serem superados para que as microalgas se tornem competitivas no mercado de biocombustíveis e como sequestradoras de $\mathrm{CO} 2$, suas potencialidades de rendimento são expressivas e, portanto, novos investimentos em P\&D são necessários.

Neste contexto, desenvolveu-se este trabalho que visa o estudo da biofixação do $\mathrm{CO} 2$ por meio do cultivo da microalga Monoraphidium sp., cultivada em fotobiorreator fechado tipo Janela, e a caracterização da biomassa microalgal produzida em relação ao: teor de lipídios totais - LT, lipídios convertíveis em biodie- 
sel - LCB, carboidratos e proteínas.

\section{MATERIAIS E MÉTODOS}

Para a realização deste trabalho, utilizou-se a cepa da microalga Monoraphidium sp. conforme figura 1, mantida na coleção do banco de culturas do Laboratório de Tecnologias Verdes (Greentec) da Escola de Química/ UFRJ.

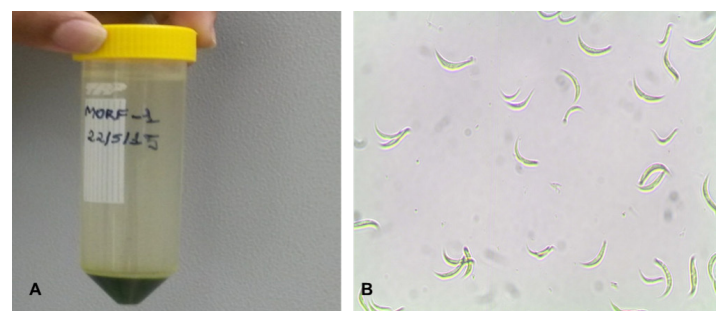

Figura 1. (A) Inóculo da cepa Monoraphidium sp.

(MORF-01); (B). Imagem da microalga observada através do microscópio óptico Olympus BX com aumento $100 \mathrm{X}$.

Escalonamento do cultivo da microalga Monoraphidium sp.

Para o escalonamento do cultivo da microalga Monoraphidium sp., foram realizadas quatro transferências (figura 2). Iniciou-se com o inoculo da cepa, previamente mantida em meio ASM-1, sob pH 7, iluminação de 170 $\mu$ fótons. $\mathrm{m}^{-2} \cdot \mathrm{s}^{-1}$ e temperatura de $23^{\circ} \mathrm{C}$, em erlenmeyer de $250 \mathrm{~mL}$ (cultivo de ativação), seguido da transferência para um segundo erlenmeyer de $2 \mathrm{~L}$ e posteriormente para um garrafão transparente de 20L. Ao fim, o cultivo do garrafão é usado como inoculo no fotobiorreator fechado tipo janela de 110L onde é monitorado o consumo de $\mathrm{CO} 2$ e o crescimento celular.
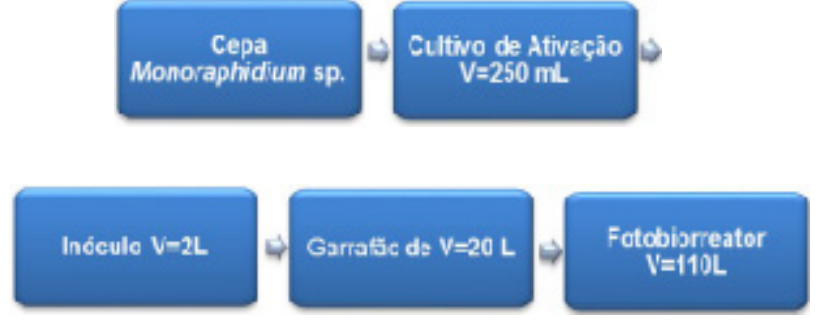

Figura 2. Etapas do escalonamento da microalga Monoraphidium sp.
Sistema de cultivo utilizado no monitoramento do consumo de CO2. Fotobiorreator de $110 \mathrm{~L}$

Para a absorção de $\mathrm{CO} 2$ pela microalga Monoraphidium sp., o cultivo foi realizado em fotobiorreator do tipo janela de 110L instalado na planta piloto do Laboratório Greentec, localizada na cobertura do Núcleo de Excelência em Petróleo e Biocombustíveis da Escola de Química/UFRJ (figura 3). Iniciou-se o cultivo em meio ASM-1; temperatura controlada entre $20-35^{\circ} \mathrm{C}$; concentração de $6,05 \times 10^{6}$ cel $\mathrm{mL}^{-1}$; injeção de ar em torno de 1 a 7L.min ${ }^{-1}$, através de um tubo de aço inox horizontal instalado no fundo do fotobiorreator, com o objetivo de aumentar a turbulência, evitando a deposição das células e aumentando o contato do líquido com o $\mathrm{CO} 2$ e sistema automatizado de controle de pH entre 7,0 a 8,0, por injeção de CO2 em três pontos do interior do fotobiorreator, usando difusores que garantem a injeção do gás em forma de micro bolhas, facilitando sua diluição na água.

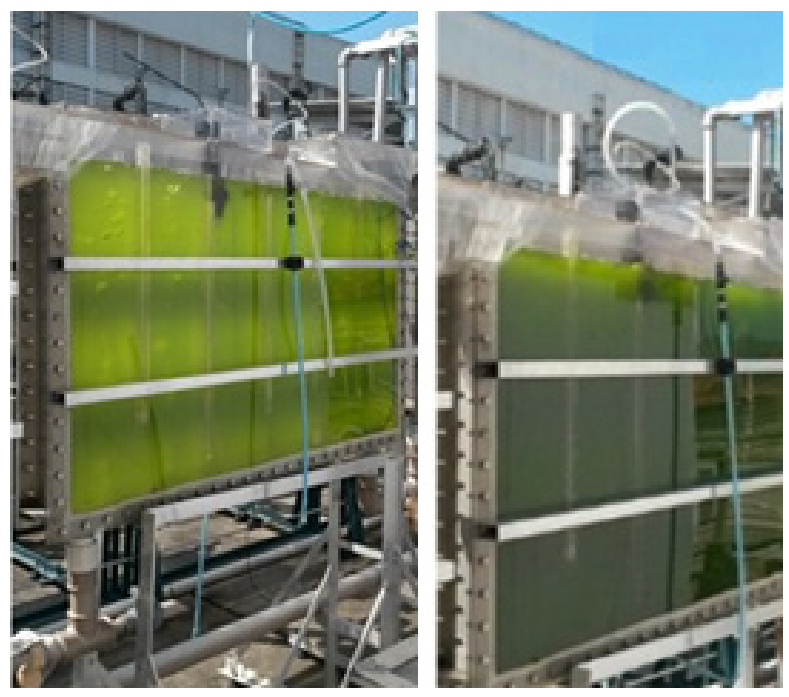

Figura 3. Cultivo da microalga Monoraphidium sp. no fotobiorreator de janela de 110L

O cultivo da microalga Monoraphidium sp. foi realizado durante um período de 12 dias, já que o foco foi medir a absorção de $\mathrm{CO} 2$. Portanto em cultivos em batelada o aumento do consumo de $\mathrm{CO} 2$ acontece nos primeiros dias de cultivo, posteriormente esse consumo come- 
ça a diminuir.

$\mathrm{O}$ monitoramento do consumo de $\mathrm{CO} 2$, considerando que é consumido nos horários em que as células estão realizando o processo de fotossíntese, foi realizado no intervalo das 8:00 às 20:00h, quantificando nesse intervalo o número de vezes em que o sistema injetava $\mathrm{CO} 2$ e o tempo de injeção. Dessa forma, como o $\mathrm{CO} 2$ sempre era injetado com vazão constante $(1 \mathrm{~L} /$ $\min ^{-1}$ ) foi possível determinar seu consumo no período monitorado. Além do consumo de $\mathrm{CO} 2$ (em $\left.\mathrm{g} / \mathrm{dia}^{-1}\right)$ outros parâmetros foram medidos diariamente durante o tempo de cultivo, como: a temperatura $\left({ }^{\circ} \mathrm{C}\right.$ ), iluminação ( $\mu$ fótons.m ${ }^{-}$ $\left.{ }^{2} \cdot \mathrm{s}^{-1}\right), \mathrm{pH}$, densidade celular $\left(\right.$ cel. $\left.\mathrm{mL}^{-1}\right)$ e biomassa seca, esta última analisada em triplicata.

O crescimento do cultivo monitorado por meio das metodologias de densidade celular e peso seco descritas a seguir:

Densidade celular: A quantidade de células (ou densidade celular, em cel. $\mathrm{mL}^{-1}$ ) presente nas amostras coletadas diariamente durante o cultivo foi determinada por contagem direta em microscópio ótico (modelo Olympus SC30) (aumento de 40 vezes), com auxílio de uma câmara de Fuchs-Rosenthal. A densidade celular foi expressa em número de células por mililitro de cultivo (cel. $\mathrm{mL}^{-1}$ ).

Determinação da biomassa seca: O peso seco foi determinado conforme a metodologia definida pelo CENPES/PETROBRAS (2013). Calcinou-se uma membrana a $575^{\circ} \mathrm{C}$ por $1 \mathrm{~h}$ para remoção de umidade; resfriou-a em dessecador e registrou-se sua massa inicial (mi); filtraram-se $10 \mathrm{~mL}$ (Vf) de cultura de microalga na membrana; em seguida, acondicionou-se a membrana em estufa a $65^{\circ} \mathrm{C}$ por $1 \mathrm{~h}$; resfrioua em dessecador e registrou-se sua massa final (mf) para determinação do peso seco e, consequentemente, dos teores de biomassa seca e de umidade, de acordo com as equações 1,2 e 3 .

Para descontar os valores de cinzas do peso seco final, as membranas com biomassa seca foram calcinadas a $575^{\circ} \mathrm{C}$ por 1 hora, res- friadas e pesadas. A determinação de peso seco foi realizada em triplicata. A figura 4 ilustra as diferentes etapas do processo.

$$
\begin{array}{ll}
\mathrm{X}=\frac{(m f-m i) \times 1000 \times \mathrm{FD}}{V f} & \text { (Equacão 1) } \\
\mathrm{TS}=\frac{\mathrm{X}}{10} & \text { (Equacão 2) } \\
\mathrm{TU}=100-\mathrm{TS} & \text { (Equação 3) }
\end{array}
$$

Onde:

$\mathrm{X}=$ concentração de biomassa ou peso seco em $\mathrm{g} / \mathrm{L}$;

$m i=$ massa da membrana calcinada sem a microalga filtrada (massa inicial);

$m f=$ massa da membrana com a microalga seca (massa final);

$V f=$ volume de cultura de microalga filtrado;

$\mathrm{FD}=$ fator de diluição;

$\mathrm{TS}=$ teor de massa seca;

$\mathrm{TU}=$ teor de umidade.

Com os dados experimentais de densidade celular, e peso seco foram elaborados gráficos em função do tempo (dia).

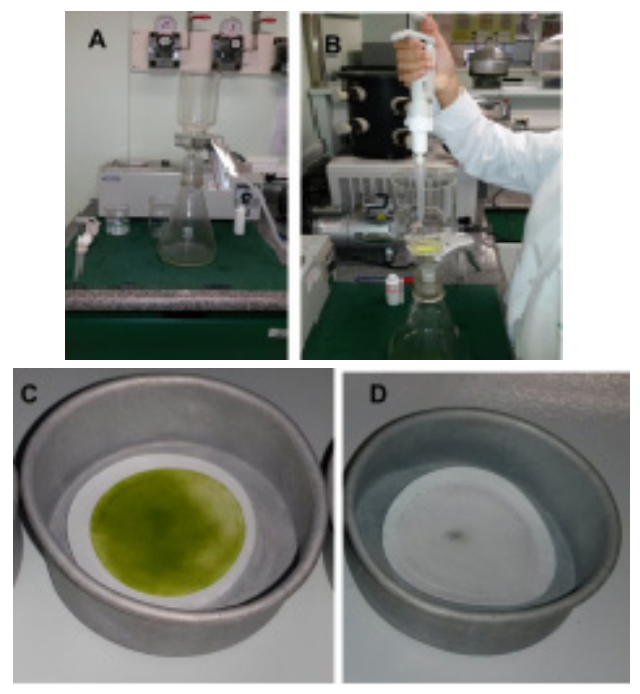

Figura 4. Determinação de peso seco. (A) Lavagem das membranas; (B) Sistema de filtração; (C) Secagem na estufa; (D) Biomassa calcinada.

Depois de 12 dias acompanhando o cultivo a biomassa foi concentrada usando centri- 
fuga Hitachi modelo CR22N. Para isso utilizou-se uma velocidade de 8.000rpm durante um tempo de 10 minutos. Para a quantificação do teor de umidade na biomassa de microalgas obtida através do processo de centrifugação, pesaram-se 2 gramas de biomassa em uma balança eletrônica Shimadzu modelo MOC63U, com temperatura de 40 a $50^{\circ} \mathrm{C}$.

Com o objetivo de determinar o teor de lipídios, carboidratos e proteínas, presentes na biomassa cultivada no fotobiorreator, a biomassa úmida de microalgas depois de centrifugada foi seca por liofilização. Esta etapa foi realizada usando liofilizador - Modelo Labconco de $4,5 \mathrm{~L}$ de capacidade, operando à temperatura de $-45^{\circ} \mathrm{C}$ e vácuo de 0,220 bar.

Para a caracterização da biomassa microalgal foram utilizadas as metodologias a seguir:

Teor de lipídios totais (LT): O teor de lipídios foi mensurado através do método de extração de lipídios totais desenvolvido por Bligh \& Dyer (1959) modificado. O processo para extração foi realizado, em triplicata, em duas etapas de acordo com o seguinte procedimento (figura 5): na primeira, a amostra foi submetida a um pré-tratamento com ácido clorídrico para hidrolisar as células; e na segunda, os lipídios foram extraídos com uma mistura de solventes (metanol/clorofórmio). Após a extração, o excesso de solvente foi removido e a quantificação dos lipídios foi realizada gravimetricamente.
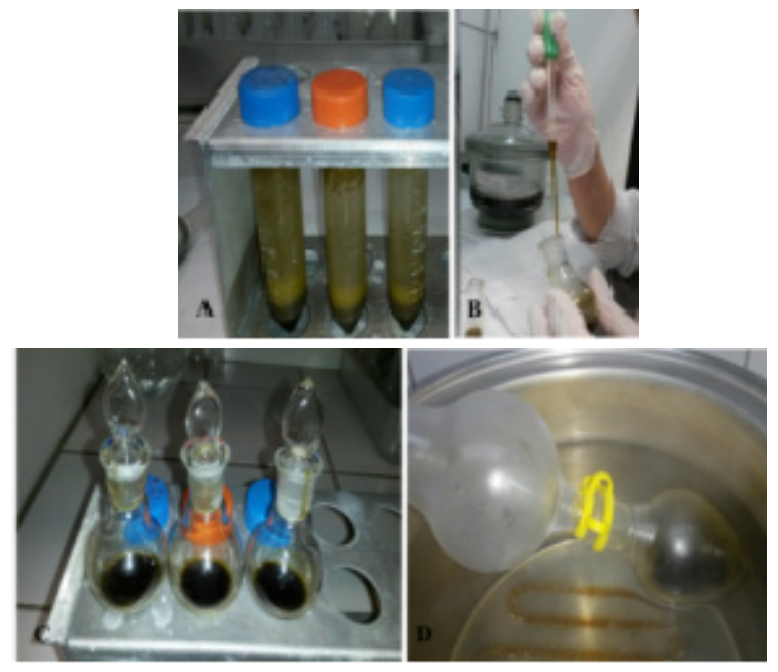

Figura 5. Processo de extração de LT (A) Pre-tratamento ácido; (B) Extração dos lipídios; (C) Amostras com lipídios; (D) Recuperação do solvente.

Teor de lipídios convertíveis em biodiesel $(L C B)$ : A determinação do teor de lipídios convertíveis em biodiesel foi realizada utilizando o método de Schmid-Bonzynski-Ratzlaff (modificado) (IDF, 1986). Baseado na digestão de biomassa de microalga com acido clorídrico, seguida da extração da fração lipídica com álcool etílico, éter etílico e éter de petróleo. Após a extração, o excesso de solvente foi removido e a quantificação dos LCB foi realizada gravimetricamente. O método foi realizado em triplicata de acordo com o seguinte procedimento (figura 6).

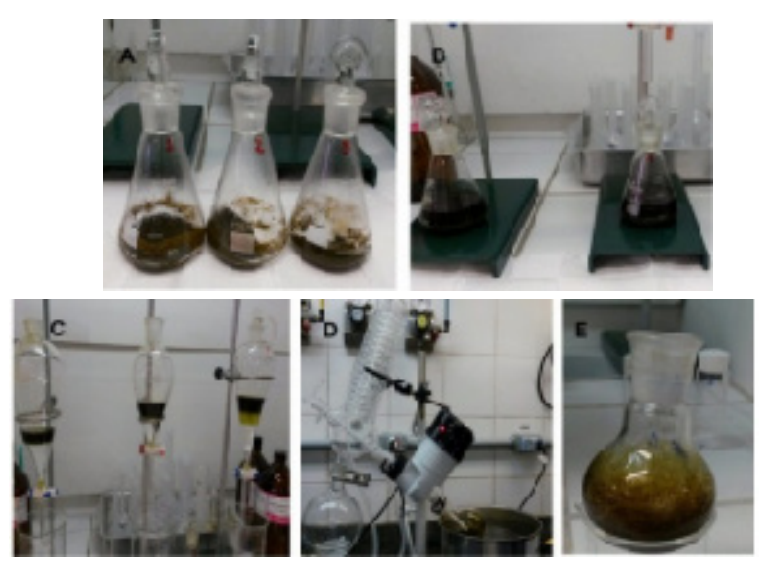

Figura 6. Processo de extração de LCB (A) Ruptura da biomassa com HCl; (B) Adição de solventes para separação da fase etérea; (C) Separação da fase etérea; (D) Rotaevaporação da fase etérea com óleo; (E) Óleo seco.

Teor de carboidratos: A extração dos carboidratos totais foi realizada segundo o método descrito por Myklestad e Haug (1972). O método denominado fenol- sulfúrico permite extrair os carboidratos solúveis em água que estão presentes na biomassa seca.

Teor de proteínas totais: A análise de proteínas totais foi realizada no Laboratório de Tecnologia de Alimentos da Escola de Química da UFRJ, pelo método de Kjeldahl clássico modificado A.O.A.C, (1995). 


\section{RESULTADOS E DISCUSSÃO}

Analisando a curva de crescimento do cultivo no fotobiorreator de Janela (figura 7) observa-se uma fase lag ou de adaptação durante os primeiros dois dias de cultivo, seguida de uma fase de crescimento exponencial que inicia a partir das 48 horas de cultivo e vai até o décimo primeiro dia, quando a concentração de células começa a diminuir. Posteriormente, depois de verificar o início da etapa de morte celular, iniciou-se a concentração do cultivo usando centrifuga Hitachi modelo CR22N.

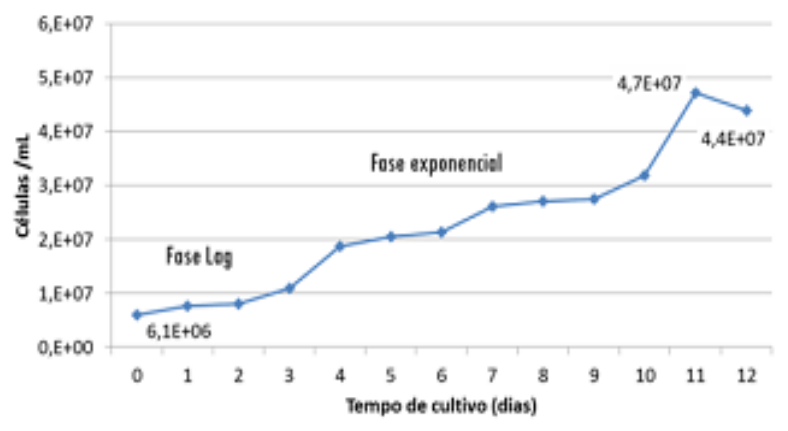

Figura 7. Curva de crescimento da microalga Monoraphidium sp. cultivada em fotobiorreator tipo Janela de 110L.

De acordo com a figura 8 o maior consumo de $\mathrm{CO} 2$ ocorreu durante os seis primeiros dias do cultivo e depois começou a diminuir. Observa-se que ao longo do cultivo o crescimento da biomassa é maior nestes seis primeiros dias. Por esta ração o cultivo foi realizado até o dia doze devido a que o foco deste trabalho foi monitorar o consumo de $\mathrm{CO} 2$ na microalga Monoraphidium sp.

Este comportamento está totalmente de acordo com os resultados de cultivos descritos na literatura (Holbrook et al, 2014), onde durante a fase de crescimento celular o processo de fotossíntese é muito mais intenso e consequentemente o consumo de $\mathrm{CO} 2$ é superior. $\mathrm{Na}$ medida em que transcorrem os dias, parte das células que estão sendo contabilizadas nas análises de contagem celular ou na determinação de peso seco não consomem $\mathrm{CO} 2$ porque estão mortas. Este comportamento é observado em cultivos em batelada. Nos cultivos em contí- nuo, como usualmente acontece em escala comercial, é possível manter o nível de absorção de CO2 sempre em níveis elevados.

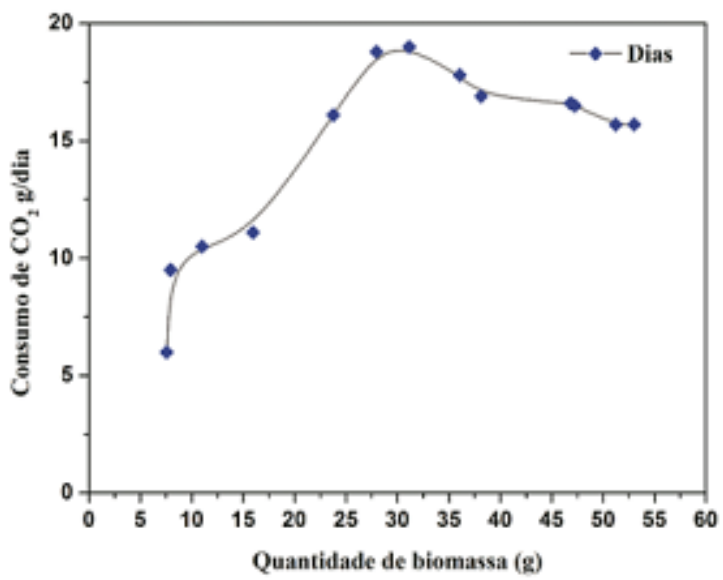

Figura 8. Quantidade de biomassa vs consumo de $\mathrm{CO} 2$ no cultivo de Monoraphidium.

Os resultados permitiram definir que, no início do cultivo (dia 0), por cada grama de biomassa produzida, estavam sendo consumidos $0,79 \mathrm{~g}$ de $\mathrm{CO} 2$. Já no último dia (dia 12), por cada grama de cultivo, foram consumidos $0,3 \mathrm{~g}$ de $\mathrm{CO} 2$. No sexto dia de cultivo, por cada grama de biomassa, foram consumidos $0,61 \mathrm{~g}$ de $\mathrm{CO}$ 2. O melhor resultado foi obtido após $24 \mathrm{~h}$ de cultivo, no qual, por cada grama de biomassa, consumiram-se aproximadamente $1,2 \mathrm{~g}$ de $\mathrm{CO} 2$.

Vale salientar que o consumo de $\mathrm{CO} 2$ foi contabilizado em um período de 12 horas, contemplando o intervalo entre 8:00 a 20:00 horas, incluindo o horário de maior incidência solar e por tanto de maior fotossínteses. O tempo de cada injeção de $\mathrm{CO} 2$ foi em média de 11 a 15 segundos.

A biomassa da microalga depois de centrifugada e liofilizada foi caracterizada seguindo os procedimentos descritos no item de materiais e métodos. Os resultados da caracterização são apresentados na tabela 1 .

Tabela 1. Composição da biomassa da microalga.

\begin{tabular}{|c|c|c|c|}
\hline$\underset{c}{\text { Protcmas }}$ & $\underset{\%}{\text { Carboidratos }}$ & $\begin{array}{l}\mathrm{L1} \\
0 \%\end{array}$ & $\underset{\%}{L C B}$ \\
\hline 42 & $32 \pm 3,37$ & $17,33 \pm 3,27$ & $8,36 \pm 2,79 \%$ \\
\hline
\end{tabular}


Neste caso, o baixo teor de LCB presente na biomassa pode estar relacionado com a composição do meio de cultura ASM-1, utilizado fundamentalmente para manutenção de cepas em escala laboratorial e consequentemente com baixa concentração de nutrientes, quando comparado com outros meios de cultivo comunmente utilizados em maior escala como BG-11 ou Bolds Basal Medium (BBM).

A partir do óleo extraído na quantificação do teor de lipídios convertíveis em biodiesel foi identificado o perfil lipídico pela cromatografia gasosa. Os resultados mostram a seguinte distribuição de ácidos graxos saturados (SAFA), monoinsaturados (MUFA) e poliinsaturados (PUFA): teor de SAFA $>$ PUFA $>$ MUFA. Dentro dos SAFA`s destaca-se o C18:0 (esteárico) em relação aos PUFA's C18:1 (Linoléico) e C18:3 (Linolênico), já o MUFA que se apresentou-se em maior quantidade foi o C18:1 (oléico).

\section{CONCLUSÕES}

Nas condições de cultivo utilizadas no fotobiorreator tipo Janela, a microalga Monoraphidium sp. mostrou-se eficiente na biofixação de $\mathrm{CO} 2$, consumindo no dia de maior crescimento celular $1,2 \mathrm{~g}$ de $\mathrm{CO} 2$ por grama de biomassa produzida.

Nos cultivos em batelada observa-se um aumento no consumo de $\mathrm{CO} 2$ apenas nos primeiros dias de cultivo, depois esse consumo começa a diminuir. Nos cultivos em contínuo, como usualmente acontece em escala comercial, é possível manter o nível de absorção de CO2 sempre em níveis elevados.

Os sistemas de cultivo de microalgas poderiam ser acoplados às chaminés de grandes indústrias emissoras de $\mathrm{CO} 2$ utilizando esse gás, resultante de processos de combustão, no processo de fotossínteses.

O CO2 extraído na etapa de purificação do biogás poderia ser injetado nos fotobiorreatores de microalgas com o objetivo de dar um destino ecológico para esse gás contaminante, responsável pelo efeito estufa.
$\mathrm{Na}$ fase de crescimento a produtividade média de biomassa no fotobiorreator tipo Janela foi de $58 \mathrm{mg} \cdot \mathrm{L}^{-1} \cdot \mathrm{dia}^{-1}$.

A biomassa de Monoraphidium sp. produzida apresentou um teor de LT de $17,37 \pm 3,27 \%$, LCB de aproximadamente $8,36 \pm 2,69 \%$, proteínas de $34,26 \pm 0,41 \%$ e carboidratos de $32 \pm 3,37 \%$. Este último poderia ser utilizado na produção de etanol via fermentação.

O teor de lipídios convertíveis em biodiesel acumulado na biomassa não foi muito elevado. Se o foco desta biomassa fosse a produção de biodiesel, as condições e meios de cultivo deverão ser modificadas visando o acumulo dos lipídios de interesse.

O perfil lipídico do óleo presente na biomassa da microalga Monoraphidium sp., cultivada no fotobiorreator tipo Janela, apresentou o seguinte comportamento: SAFA $>$ PUFA $>$ MUFA. Destacando-se o SAFA C18:0 (esteárico), os PUFA`s C18:1 (Linoléico) e C18:3 (Linolênico) e o MUFA C18:1 (oléico).

\section{REFERÊNCIAS}

ASSOCIATION OF OFFICIAL ANALYTICAL CHEMISTS - AOAC. 1995. Official methods of analysis. 16 ed. Arlington: AOAC, v. 1.

BRENNAN, L.; OWENDE, P. 2010. Biofuels from microalgae - a review of technologies for production, processing, and extractions of biofuels and co-products. Renewable and sustainable energy reviews, v. (14), n. 2, p. 557-577.

BLIGH, G. AND DYER, W. 1959 A Rapid Method for Total Lipid Extraction and Purification. Canadian Journal of Bio- chemistry and Physiology, 37, 911-917. http://dx.doi. org/10.1139/059-099.

EBC.http://www.ebc.com.br/noticias/economia/2013/02/fabrica-de-biodiesel-vai-suprirdemanda-pelo-combustivel-no-rio. Acesso em: Janeiro de 2017. 
GREENWELL, H. C. et al. 2010. Placing microalgae on the biofuels priority list: a review of the technological challenges. Journal of Royal Society Interface. v. 7, p. 703-726.

HOLBROOK, GABRIEL P., ZACHARY DAVIDSON, ROBERT A. TATARA, NORBERT L. ZIEMER, KURT A. ROSENTRATER, AND W. SCOTT GRAYBURN. 2014. "Use of the Microalga Monoraphidium Sp. Grown in Wastewater as a Feedstock for Biodiesel: Cultivation and Fuel Characteristics." Applied Energy 131: 386-93. doi:10.1016/j.apenergy.2014.06.043.

MATA TM, MARTINS AA, CAETANO NS. 2010. "Microalgae for Biodiesel Production and Other Applications: A Review." Renew Sust Energ Rev, 14:217-32.

MYKLESTAD, S. \& HAUG, A. 1972. Production of carbohydrates by the marine Chaetoceros affinis var. O Willei (Gran.) Hustedt. I. Effect of the concentration of nutrients in the culture medium. J. exp mar. Biol. Ecol (9):125-136.

PERLINGEIRO G, CARLOS AUGUSTO. 2014. Biocombustíveis No Brasil: Fundamentos, Aplicações e Perspectivas. Synergia 241247.

RAVEN H, PETER H, RAY F, EICHHORN SE. 2010. "Biology of Plants." 7 thed. Ne: 124-27.

REPORT, ANNUAL. 1987. "FY 1986 Aquatic Species Program".

SCHENK, P. M, THOMAS-HALL,S. S. R., STEPHENS, E., MARX, U. C, MUSSGNUG, J. H., POSTEN, C., KRUSE, O. \& HANKAMER, B., 2008. Second Generation Biofuels:High-Efficiency Microalgae for Biodiesel Production. Bioenergy. Res.1:20-43.

SHRIVASTAV, ANUPAMA, SANJIV K MISHRA, WILLIAM I SUH, WASIF FAROOQ, MYOUNGHOON MOON, TAE-HYOUNG KIM, KANHAIYA KUMAR, GANG-
GUK CHOI, MIN S PARK, AND JI-WON YANG. 2015. "Characterization of Newly Isolated Oleaginous Microalga Monoraphidium sp. for Lipid Production under Different Conditions." ALGAL 12. Elsevier B.V.: 289-94. doi:10.1016/j.algal.2015.08.015.

SCHMID-BONDZYNSKI-RATZLAFF. 2012. Extração e quantificação de lipídios em microalgas. Modificado pela Universidade Federal de Viçosa. Departamento de Tecnologia de Alimentos. 